\title{
Reconsidering Batteries in Energy Harvesting Sensing
}

\author{
Neal Jackson \\ neal.jackson@berkeley.edu \\ University of California, Berkeley
}

\author{
Joshua Adkins \\ adkins@berkeley.edu \\ University of California, Berkeley
}

\author{
Prabal Dutta \\ prabal@berkeley.edu \\ University of California, Berkeley
}

\begin{abstract}
For the past decade, the status-quo for energy harvesting sensors has been to buffer small amounts of energy in capacitors to intermittently work through a sensing task. While using capacitors for storage offers these systems indefinite lifetime, it comes at a cost - they must tolerate the decreased availability, lower energy utilization, and more complex programming models inherent to a volatile, intermittent design. We argue that many of these problems stem from insufficient energy storage and could be eliminated with the use of batteries. Recent advances in rechargeable battery technology weaken the historical arguments against their use. We believe that using batteries in energy harvesting sensors will push us closer to a class of reliable, general purpose devices that can better serve human-centric sensing applications than their capacitor-based counterparts at the cost of having a finite, but long, lifetime.
\end{abstract}

\section{CCS CONCEPTS}

- Hardware $\rightarrow$ Sensors and actuators; Batteries; Reusable energy storage; Energy distribution; • Software and its engineering $\rightarrow$ Power management;

\section{KEYWORDS}

Energy Harvesting, Sensor, Battery, Capacity

\section{INTRODUCTION}

The first sensor network nodes relied on non-rechargeable batteries (primary cells) for energy storage [27, 33]. These primary cells provided reliable power that enabled quick initial tests and deployments. However the drawbacks of this design quickly became clear-the short sensor node lifetimes achievable with reasonably sized primary cells required frequent battery replacement for nodes to remain operational, and as a result, researchers started looking to energy harvesting as an alternative power source. This began with outdoor solar harvesting, but soon transformed as researchers started to leverage lower power components and new programming models to enable energy harvesting sensor nodes with significantly less capable power supplies.

Starting with directed, RFID-based sources [42], and moving to less reliable energy sources like indoor solar and vibration [6, 13, 47], these designs fueled a vision of perpetually operational sensor nodes powered by the ambient environment. At the time, capacitors

Permission to make digital or hard copies of part or all of this work for personal or classroom use is granted without fee provided that copies are not made or distributed for profit or commercial advantage and that copies bear this notice and the full citation on the first page. Copyrights for third-party components of this work must be honored.

For all other uses, contact the owner/author(s).

ENSsys'18, November 4, 2018, Shenzhen, China

(c) 2018 Copyright held by the owner/author(s)

ACM ISBN 978-1-4503-6047-0/18/11.

https://doi.org/10.1145/3279755.3279757 were the only easy, off-the-shelf method of storing energy from these ultra-low current power sources. Supercapacitors had high leakage that could waste a significant portion of the already limited energy. Lithium batteries were difficult to manage, as low power battery management and charging ICs were not readily available. Additionally the theoretically infinite lifetime of capacitors further supported the goals of perpetual sensing, especially compared to the life-cycle limits of batteries. Over time, other weaknesses of batteries compounded the arguments, including their temperature sensitivity, physically large form factor, and potential danger for both the user and the environment.

Unfortunately, capacitors offer very low energy density, often much less than $1 \mathrm{Wh} / \mathrm{L}$ (compared to $100 \mathrm{Wh} / \mathrm{L}$ for a small LiPo cell), and devices that use them must cope with the limitations this presents. These include lower energy utilization (if a device could harvest but the capacitor is full), reduced availability (a sensor cannot perform a required task because its capacitor is already depleted), decreased responsiveness (if the capacitor does not have energy to detect and respond to an event), and the inability to easily perform high-energy tasks (if the capacitor does not have enough total energy storage for task completion).

Many solutions have been proposed to tackle these problems. Software checkpointing and associated programming models that ease their use allow relatively high-energy tasks to be completed correctly [26]. The introduction of wakeups based on the sensed phenomena decreases the probability of missing an event [6]. Federating energy stores for each predefined component workloads decreases latency between tasks and eases modularity [17]. Reconfigurable energy buffer sizing lowers inter-task latency for arbitrary workloads [9]. Even with these solutions, however, it is still not easy to program and use capacitor-based energy harvesting sensors, and they still have decreased availability and energy utilization compared to a solution with larger energy storage capacity.

While capacitors may have been the best choice for energy harvesting sensors a decade ago, this decision should be reevaluated in the face of new, modern technology. We consider the changes in battery chemistries and associated battery management ICs, and we argue that for modern sensor designs, a power supply that offers an indefinite lifetime may not justify the cost of low energy capacity. We believe that new battery technologies address many of the original assumptions that limited their early adoption, and while they do not provide theoretically infinite lifetimes, nor will they operate in all sensing scenarios, they may be a better choice for the vast many common sensing tasks. It is time to reconsider the use of batteries in ultra-low power energy harvesting sensors.

\section{IS IMMORTALITY WORTH THE PRICE?}

Through the wealth of work towards making intermittent systems easier to program and more reliable, researchers have implicitly made the argument that a power supply with an indefinite lifetime, 
extreme temperature resistance, and small physical size is worth the costs of significantly reduced energy capacity. This continually growing body of work also serves as evidence that using and deploying systems with this reduced energy capacity is neither easy nor a sufficiently solved problem.

Developing programs that can continue forward progress in the face of volatile energy availability is a complex and headacheinducing experience. In situations of energy drought, intermittent systems will deplete their small capacitor energy stores, and lacking energy, they power off and lose state, potentially in the middle of an important operation and for an extended period of time. In the worst case, the device will be stuck in a Sisyphean loop of starting up and dying before ever finishing a task. To alleviate some of this pain, researchers have developed tools and language primitives to assist with writing intermittent applications [20,26], but the reality remains: these systems do not have enough storage to withstand absences of harvestable energy and must micro-manage the little energy available to them to ensure forward progress.

At its core, using a small energy buffer severely impacts energy utilization, because smaller storage capacity is more likely to fill up even when harvestable energy is still available. In turn, this lowers availability, in terms of both uptime and responsiveness. An intermittent occupancy sensor may fail to perform detection at night, and miss the first person arriving in a space the following day. While the capacity of supercapacitors may allow an intermittent sensor to survive overnight, it will not survive longer energy droughts of a few days to a week.

Another symptom of small energy storage includes the inability to perform complex and long-running tasks like cryptographic operations or firmware updates in a timely manner. These operations are severely limited because they cannot be completed in one iteration and must make intermittent progress. Additionally, storage capacity creates a limit on the kinds of sensors that can be supported. With capacitors for storage, it is very unlikely that sensing modalities that require substantial and sustained energy for operation, like heated humidity sensors and cameras, will operate, even at low duty cycles.

These systems are also forced to micromanage their energy state on the order of milliseconds [26]. While tools exist to aid in this, users must write applications that are aware of minutiae of energy availability. This complicates applications and limits the generality of both the application and sensing platform for different use cases. The Flicker platform explored enabling more generality by federating the capacitor storage among components, but its applications must still be programmed with specific knowledge of energy availability [18]. By increasing energy storage, we can instead manage energy over the course of days, weeks, or even months, and even have the capacity for occasional high power tasks. Dilating the timing requirements for energy management makes techniques like checkpointing and complex hardware reconfiguration unnecessary. Simple software energy policies that dynamically adjust workload intensity become possible and sufficient. Being powered for long periods has the added benefit of saving energy by significantly reducing the number of system reboots [18]. The system can instead remain in an ultra low power sleep state between operations and in periods of low energy availability.

\subsection{Considering a Holistic Lifetime}

In addition to considering the lifetime of a sensor's power supply, we should also note that other components of a sensor have a finite lifetime, which may render the goal of an indefinite lifetime moot. Most notably, some components exhibit significant long-term calibration drift. For example, each year a humidity sensor [41] expects a quarter of a percent relative humidity drift, while an oscillator [45] expects $3 \mathrm{ppm}$ drift. There is also the question of relevancy in the face of decades of future progress in networking and security. At some point, the sensors we build today will be obsolete, regardless of their theoretical lifetimes. Instead of indefinite sensors, we need sensors that last long enough and provide enough benefit to justify their eventual and inevitable replacement.

\section{BATTERIES: NEW AND IMPROVED}

Rechargeable batteries are the obvious high-capacity energy storage option to avoid the pains that come from a small energy buffer. Many intermittent systems papers, however, dismiss them as expensive [17-20], short-lived [9, 17-20, 26, 47]. temperature-sensitive [9, 17-20, 26], less efficient [17-20], bulky [17-20, 47], and dangerous [17-20]. In this section, we re-examine each of these arguments, in the context of newly available modern battery chemistries, including Lithium Titanate (LTO) and Lithium Iron Phosphate $\left(\mathrm{LiFePo}_{4}\right)$, and low power battery management ICs that simplify energy harvesting designs [44], concluding that these new chemistries do not exhibit the same detracting qualities as those available a decade ago. Batteries still underperform in some metrics compared to ceramic and tantalum capacitors, but their orders of magnitude increase in energy density and storage capacity likely justify these costs.

Expensive to Buy. As seen in Table 1, small, 2-40 mAh LTO batteries can be found for $\$ 6.75$ USD each from US distributors and $\$ 1.01$ USD each from Chinese distributors, even in small quantities $[21,22]$. While greater than the sub-dollar cost of the several tantalum and ceramic capacitors that comprise a capacitor storage bank [3,35], these prices are comparable to supercapacitors $[23,30,38]$, and the cost of other key components in an energy harvesting system, like the MCU [31], harvester [36] and sensors [41], which cost around \$5 USD each in low quantities. A battery will not constitute the driving cost of developing a sensor that integrates one. We expect prices for batteries to continue to drop with increased usage, distribution, and scale.

Short-Lived. While it is true that the capacity of rechargeable batteries degrades as a function of cycle count and the depth-ofdischarge (DoD), new technologies and defensive design can mitigate this problem. LTO and $\mathrm{LiFePo}_{4}$ cells can both withstand 4-10x more cycles than traditional LiPo cells before experiencing similar capacity degradation. In experiments, LTO cells were found to experience 3400 full cycles before noticeable capacity degradation at room temperature [15], and available commercial cells claim 7000 cycles at $0.5 \mathrm{C}$ charge/discharge rate [22]. Similarly, $\mathrm{LiFePo}_{4}$ cells have been observed to withstand between 3000 and 4000 cycles before degradation $[32,37,46]$. Additionally, lowering DoD to $10 \%$ exponentially decreases the rate of capacity loss, resulting in potential lifetimes of greater than 10,000 cycles before reaching $80 \%$ 


\begin{tabular}{|c|c|c|c|c|c|c|c|c|c|c|}
\hline \multirow{2}{*}{ Technology } & \multirow{2}{*}{ Capacity } & \multirow{2}{*}{$\begin{array}{l}\text { Volume } \\
\left(\mathrm{mm}^{3}\right)\end{array}$} & \multirow{2}{*}{$\begin{array}{l}\text { Energy Density } \\
(\mathrm{Wh} / \mathrm{L})\end{array}$} & \multirow{2}{*}{$\begin{array}{l}\text { Temperature Range } \\
\left(\text { Charge/Discharge }{ }^{\circ} \mathrm{C}\right)\end{array}$} & \multirow{2}{*}{$\operatorname{ESR}(\Omega)$} & \multirow{2}{*}{$\begin{array}{l}\text { Self-Discharge } \\
\text { (nA) }\end{array}$} & \multicolumn{2}{|c|}{ Cycle Life (Cycles) } & \multicolumn{2}{|c|}{ Cost (USD) } \\
\hline & & & & & & & $100 \% \mathrm{DoD}^{1}$ & $10 \% \mathrm{DoD}^{1}$ & US $^{\circ}$ & $\mathrm{China}^{\mathrm{O}}$ \\
\hline \multirow[t]{2}{*}{ MLCC } & $47 \mu \mathrm{F}[34]$ & $1.28^{\mathrm{a}}$ & $0.046^{b}$ & $-55-125$ & $0.001-0.1 \mathrm{~g}$ & $<10^{\mathrm{i}}$ & Inf. $\mathrm{m}$ & Inf. ${ }^{\mathrm{m}}$ & 0.16 & 0.03 \\
\hline & $100 \mu \mathrm{F}[35]$ & $9.2^{\mathrm{a}}$ & $0.013^{\mathrm{b}}$ & $-55-125$ & $0.001-0.1 \mathrm{~g}$ & $<10^{\mathrm{i}}$ & Inf. $\mathrm{m}$ & Inf. $\mathrm{m}$ & 0.31 & 0.04 \\
\hline \multirow[t]{2}{*}{ Tantalum } & $100 \mu \mathrm{F}[3]$ & $9.2^{\mathrm{a}}$ & $0.013^{b}$ & $-55-85$ & 0.2 & $<10^{\mathrm{i}}$ & Inf. ${ }^{m}$ & Inf. ${ }^{m}$ & 0.28 & 0.17 \\
\hline & $220 \mu \mathrm{F}[3]$ & $9.2^{\mathrm{a}}$ & $0.027^{b}$ & $-55-85$ & 0.07 & $<10^{\mathrm{i}}$ & Inf. ${ }^{m}$ & Inf. ${ }^{m}$ & 0.37 & 0.16 \\
\hline \multirow[t]{3}{*}{ Supercapacitor } & $7.5 \mathrm{mF}[38]$ & 7.2 & $0.83^{\mathrm{c}}$ & $-30-70 \mathrm{~d}$ & 25 & - & $>10000$ & - & 2.42 & - \\
\hline & $100 \mathrm{mF}[23]$ & 1128 & $0.07^{\mathrm{c}}$ & $-25-70^{d}$ & 100 & $<10^{\mathrm{j}}$ & - & - & 1.10 & - \\
\hline & $470 \mathrm{mF}[30]$ & 940 & $0.62^{\mathrm{b}}$ & $-40-70^{\mathrm{d}}$ & 45 & $<1000$ & $100000+/ 4 \mathrm{yr}[29]^{\mathrm{n}}$ & $-{ }^{\mathrm{n}}$ & 5.06 & 1.00 \\
\hline \multirow[t]{3}{*}{ LiPo } & $37 \mathrm{mWh}[12]$ & 297 & 125 & $0-40 /-20-60 \mathrm{e}$ & $-\mathrm{k}$ & $30-100[48]$ & $300-500$ & $10000+[14,28]$ & - & 0.80 \\
\hline & $148 \mathrm{mWh}[10]$ & 660 & 224 & $0-40 /-20-60 \mathrm{e}$ & 0.1 & $120-400[48]$ & 300 & $10000+[14,28]$ & 4.50 & 0.51 \\
\hline & $148 \mathrm{mWh}[2]$ & $1005^{\mathrm{a}}$ & 224 & $0-40 /-20-60 \mathrm{e}$ & 3 & $120-400[48]$ & 500 & $10000+[14,28]$ & 1.62 & - \\
\hline \multirow[t]{2}{*}{ LTO } & $4.3 \mathrm{mWh}[22]$ & 88 & 49 & $-35-70$ ef & 8 & $-\mathrm{k}$ & 2000 & $10000+[15]$ & - & 1.01 \\
\hline & $60 \mathrm{mWh}[21,22]$ & 685 & 88 & $0-40 /-20-60$ ef & 2.4 & $-\mathrm{k}$ & 2000 & $10000+[15]$ & 6.75 & 1.01 \\
\hline $\mathrm{LiFePo}_{4}$ & $96 \mathrm{mWh}[40]$ & 1020 & 94 & $0-40 /-20-60 \mathrm{e}$ & 0.1 & $160[43]$ & 2000 [39] & $30000+[32,37,46]$ & - & 0.95 \\
\hline \multicolumn{11}{|c|}{$\begin{array}{l}\text { a Standard packages in order of increasing volume: } 0603,1206,2032, C R 123 \mathrm{~A} . \quad \mathrm{b} \text { Assumed } 3 \mathrm{~V} \text { for energy calculation. } \\
\mathrm{d}_{\text {Supercapacitors experience higher ESR at lower temperatures and higher leakage at higher temperatures [29]. }} \\
e_{\text {Lithium batteries experience higher ESR, higher leakage, lower capacity and shorter lifetimes at temperature extremes. }}\end{array}$} \\
\hline \multicolumn{11}{|c|}{$\mathrm{f}_{\text {We believe these are produced by the same manufacturer, but have different suppliers and datasheets. We are skeptical of the wide temperature range. }}$} \\
\hline $\mathrm{g}_{\mathrm{ESR}}$ is freque & dependent. $h_{1}$ & is conservati & alculated into rated & [11]. $\quad \mathrm{i}$ Both tested an & ulated from in & ation resistance af & sorption period. & & & \\
\hline
\end{tabular}

Table 1: A comparison of energy storage technologies that may be used on sensor nodes. Data is based on specific components and their datasheets, and we attempt to choose representative components for each category, however some technologies such as supercapacitors are rapidly evolving. Other citations point to capabilities of the storage technologies not specified by their datasheets. We find that for most applications lithium-based batteries provide much higher energy density without reasonably impacting sensor lifetime, cost, or function. The minority of sensing applications, such as those operating at extreme temperatures, may require capacitors until battery technologies improve.

of rated capacity with $\mathrm{LiFePo}_{4}[32,46]$. We also expect exponential gains for LTO cells with reduced DoD and estimate an order of magnitude increase in cycle lifetime. These cycle estimations hold true even for relatively high temperatures $\left(60^{\circ} \mathrm{C}\right)$ [46]. LTO chemistries can be expected to survive one thousand cycles at $100 \%$ $\mathrm{DoD}$ at $55^{\circ} \mathrm{C}$ [16]. Life cycle expectations for both $100 \%$ and $10 \%$ DoD are summarized in Table 1.

With the energy capacity provided by batteries, we expect cycles that are slower than diurnal, implying 20-50 years or more of function before observing significant capacity reduction with observed cycle lifetimes. Supercapacitors also experience lifetime issues that are tied to their operational hours rather than number of cycles. Some datasheets predict as few as four years before reaching $80 \%$ of rated capacity under standard indoor environmental conditions and $3 \mathrm{~V}$ storage [30]. While this may not be true of all supercapacitors, it is not clear that they provide advantages in lifetime over batteries without further testing.

Additionally, LTO chemistries have been shown to exhibit no long-term cell damage when undervolted, even to zero volts [5]. This is a significant improvement more traditional technologies like LiPo which would suffer capacity degradation if undervolted in cases of long storage or absence of charging, a common occurrence for energy harvesting systems.

Low Efficiency. Low efficiency of an energy store is caused by high or increasing equivalent series resistance (ESR) and self-discharge. Power losses due to ESR can primarily be attributed to high current events, such as a radio transmission. We find the ESR of small batteries is higher than that of ceramic and tantalum capacitors, however it is slightly better than the ESR of supercapacitors. With the capacitors and batteries mentioned in Table 1 , an $8 \mathrm{~mA}$ radio transmission from a steady $3 \mathrm{~V}$ would incur less than $0.06 \%$ in resistive losses from a tantalum capacitor, $2.1 \%$ losses from an LTO battery, and $6.6 \%$ losses from an supercapacitor.

The self-discharge of batteries is dependent on their capacity and environmental factors, however for small batteries in standard conditions we expect 30-500 nA of equivalent self-discharge current. This is much higher than the self discharge of ceramic and tantalum capacitors, but similar to larger supercapacitors even after their absorption period, which may last several hundred hours and have up to 10x the self-discharge current [29]. Batteries may still not be suitable for sensors that expect very low harvesting currents.

Temperature Sensitive. Batteries are more temperature sensitive than other forms of energy storage, but they are improving. Some datasheets and authors report operating batteries successfully as low as $-30^{\circ} \mathrm{C}$ and as high as $75^{\circ} \mathrm{C}[1,8,22]$, however, the capacity, ESR, and cycle lifetimes of a battery will still degrade at extreme temperatures $[43,46]$, and further study of these results is warranted. We note that supercapacitors also experience issues such as drastically increased ESR at very low temperatures [29]. Rated temperature ranges for discussed technologies are summarized in Table 1.

Even with these temperature limitations, we believe that most deployment scenarios for energy harvesting sensor nodes do not exceed the temperature ranges of current battery technology, including nearly all indoor sensor deployments and many outdoor deployments.

Bulky. As presented in Table 1, small batteries are 50-500x more dense than the supercapacitors and three to five orders of magnitude more dense than ceramic and tantalum capacitors. Even with an order of magnitude capacity reduction to achieve high cycle lifetimes, batteries have a usable energy density of 5-50x greater than supercapacitors of similar physical size. The batteries shown 


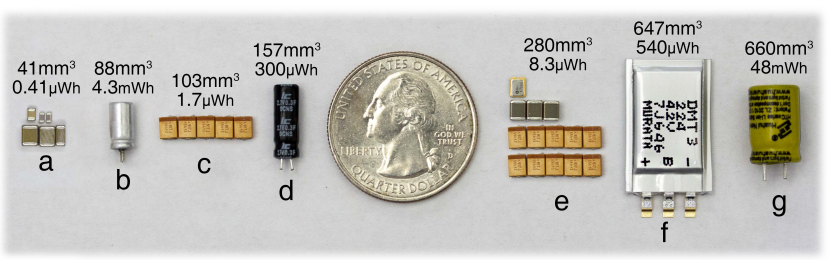

Figure 1: A size comparison of energy storage methods including capacitors, supercapacitors, and batteries. They are ordered left to right, by their (total) volume. Total volumes are listed above the respective device. Configuration (a), (c) and (e) represent the energy storage configurations used in the Flicker platfrom with BLE and several sensors [18], the Solar Monjolo [6] and the Capybara temperature monitor and alarm [9], which have total capacitances and energy capacities of $119 \mu \mathrm{F}(0.41 \mu \mathrm{Wh}$ at $5 \mathrm{~V})$, $500 \mu \mathrm{F}(1.7 \mu \mathrm{Wh}$ at $5 \mathrm{~V})$ and $8.8 \mathrm{mF}(8.3 \mu \mathrm{Wh}$ at $2.6 \mathrm{~V})$, respectively. Capacitors (d) [7] and (f) [30] are large supercapacitors available on the Capybara platform and have the capacitances and energy capacities of $300 \mathrm{mF}(300 \mu \mathrm{Wh}$ at $2.7 \mathrm{~V})$ and $220 \mathrm{mF}(540 \mu \mathrm{Wh}$ at $4.2 \mathrm{~V}$ ) respectively. While all pictured devices are similar in size and appearance, (b) and (g) are actually small LTO battery cells with $1.8 \mathrm{mAh}(4.3 \mathrm{mWh}$ at $2.4 \mathrm{~V})$ and $20 \mathrm{mAh}(48 \mathrm{mWh}$ at $2.4 \mathrm{~V})$ capacity respectively [22]. The LTO battery (b) is the second smallest of all configurations of energy storage presented here and also provides an order of magnitude more energy capacity compared to (f), the largest supercapacitor presented.

Figure 1 are as small as $88 \mathrm{~mm}^{3}$, and resemble small through-hole capacitors. Battery (b) is smaller in volume than many of the capacitor configurations presented in the literature, only outdone by systems like Flicker (a) which store energy in only a few ceramic capacitors [18], and offers an order of magnitude more energy storage than the largest supercapacitor presented. When considering the size of other components in the system, most notably the harvester (solar panel, thermocouple, or piezoelectric device), the combination of large ICs, and large sensors like a PIR motion sensor, the size of existing small rechargeable batteries is not significant. Even the smallest energy harvesting sensor system to our knowledge, the Michigan Micro Mote [25], is on the scale of a capacitor and utilizes a thin-film battery for energy storage.

Dangerous. Traditional lithium metal batteries, such as lithium cobalt chemistries, present risk of fire and release of toxic gas under electrical and mechanical stress. Newer lithium-based chemistries such as $\mathrm{LTO}$ and $\mathrm{LiFeP}_{4}$ are considered much safer, as they exhibit less thermal runaway under electrical, mechanical, and thermal abuse [4, 24]. Additionally, unlike traditional lithium chemistries, LTO has been shown to not leak any toxic and reactive gasses in the case of thermal abuse [4]. They only release noble gasses. While there is potential for danger under abuse conditions of these batteries, there is ongoing research to create safer lithium batteries [24].

\section{DISCUSSION}

For applications that involve extreme temperatures, the high possibility of mechanical damage, and some scenarios that entirely prevent any sensor replacement, we expect that capacitor-based intermittent systems remain the best and perhaps only option. These include applications such as simple and inexpensive satellites that must exist in the cold of space, sensors embedded in concrete that are expected to monitor buildings over decades, or sensors meant to monitor inside of the human body. While there are some methods to make batteries function in these environments and ongoing efforts to continue to improve the properties of batteries such that they might be sufficient for these applications, these are the places in which a capacitor-based energy store has a great advantage.

We would like to reemphasize however that many applications do not take place in these conditions. The instrumentation of our homes, offices, factories and most of our outdoor spaces will not stress the properties of batteries and cause them to fail. These spaces will be occupied by people who can replace sensors at a rate similar to the rate at which we replace the rest of our furniture, appliances, and technology. Most importantly, these human-facing applications will see large benefits from the increase in availability and responsiveness that batteries have the potential to provide.

\section{CONCLUSIONS}

After a decade of designing intermittent systems and tackling their issues, it is time to reevaluate the necessity of capacitor-based storage in the face of technology improvements. New, modern battery technologies and management techniques allow for high capacity, long lifetime sensor power supplies without the costs commonly associated with older battery chemistries. We believe their integration into sensor designs will lead to more capable devices with greater availability. Battery-based sensors will ultimately enable dense deployments and autonomous applications in our occupied environment.

\section{ACKNOWLEDGMENTS}

This work was supported in part by the CONIX Research Center, one of six centers in JUMP, a Semiconductor Research Corporation (SRC) program sponsored by DARPA, and with support from the Building Technology Urban Systems group at Lawrence Berkeley National Laboratory (LBNL). Additionally, this material is based upon work supported by the National Science Foundation Graduate Research Fellowship Program under grant number DGE-1106400.

\section{REFERENCES}

[1] A123 Systems. 2012. Nanophosphate High Power Lithium Ion Cell. http:// www.batteryspace.com/prod-specs/6610.pdf. (2012).

[2] AA Battery Corp. 2006. Rechargeable Li-ion Button Battery. http://www. batteryspace.com/productimages/aa/20060224/LIR2032_new1.pdf. (2006).

[3] AVX. 2018. TPS Series Capacitor Datasheet. http://datasheets.avx.com/TPS.pdf. (2018).

[4] Ilias Belharouak, Gary M. Koenig, and K. Amine. 2011. Electrochemistry and safety of Li4Ti5O12 and graphite anodes paired with LiMn2O4 for hybrid electric vehicle Li-ion battery applications. Fournal of Power Sources 196, 23 (2011), 10344 - 10350. DOI : https://doi.org/10.1016/j.jpowsour.2011.08.079

[5] Michael Brunell, Brad Hanauer, Melanie Loveridge, Richard Dashwood, and Rohit Bhagat. 2016. Effect of Zero Volt Storage on Commercial Lithium Titanate Cells. In Meeting Abstracts. The Electrochemical Society, 896-896.

[6] Bradford Campbell and Prabal Dutta. 2014. An Energy-harvesting Sensor Architecture and Toolkit for Building Monitoring and Event Detection. In Proceedings of the 1st ACM Conference on Embedded Systems for Energy-Efficient Buildings (BuildSys '14). ACM, New York, NY, USA, 100-109. DOI : https://doi.org/10.1145/ 2674061.2674083 
[7] Illinois Capacitor. 2017. DCN Super Capacitors. https://www.illinoiscapacitor com/pdf/seriesDocuments/DCN\%20series.pdf. (2017).

[8] Kebin Chen, Zhiqiang Yu, Shawn Deng, Qiang Wu, Jianxin Zou, and Xiaoqin Zeng. 2015. Evaluation of the low temperature performance of lithium manganese oxide/lithium titanate lithium-ion batteries for start/stop applications. Fournal of Power Sources 278 (2015), 411 - 419. DOI : https://doi.org/10.1016/j.jpowsour.2014. 12.051

[9] Alexei Colin, Emily Ruppel, and Brandon Lucia. 2018. A Reconfigurable Energy Storage Architecture for Energy-harvesting Devices. In Proceedings of the Twenty-Third International Conference on Architectural Support for Programming Languages and Operating Systems (ASPLOS '18). ACM, New York, NY, USA, 767781. DOI: https://doi.org/10.1145/3173162.3173210

[10] Data Power Technology. 2015. Polymer Li-ion Rechargeable Battery Specification. https://cdn.sparkfun.com/datasheets/Prototyping/SPE-00-301120-40mahen-1.0ver.pdf. (2015).

[11] L. M. Feeney, C. Rohner, and A. Lindgren. 2014. How do the dynamics of battery discharge affect sensor lifetime?. In 2014 11th Annual Conference on Wireless On-demand Network Systems and Services (WONS). 49-56. DOI : https://doi.org/ 10.1109/WONS.2014.6814721

[12] General Electronics Technology Shenzhen. 2018. Ultrathin LiPo Battery Cell. https://www.alibaba.com/product-detail/Ultrathin-lipo-rechargeablebattery-cell-181115_60683655333.html?spm=a2700.7724857.main07.67. 25bc7633CwPhhF. (2018).

[13] M. Gorlatova, T. Sharma, D. Shrestha, E. Xu, J. Chen, A. Skolnik, D. Piao, P. Kinget, J. Kymissis, D. Rubenstein, and G. Zussman. 2010. Prototyping Energy Harvesting Active Networked Tags (EnHANTs) with MICA2 Motes. In 2010 7th Annual IEEE Communications Society Conference on Sensor, Mesh and Ad Hoc Communications and Networks (SECON). 1-3. DOI : https://doi.org/10.1109/SECON.2010.5508227

[14] T. Guena and P. Leblanc. 2006. How Depth of Discharge Affects the Cycle Life of Lithium-Metal-Polymer Batteries. In INTELEC 06 - Twenty-Eighth International Telecommunications Energy Conference. 1-8. DOI : https://doi.org/10.1109/ INTLEC.2006.251641

[15] Florian Hall, Jonas Touzri, Sabine Wußler, Hilmi Buqa, and Wolfgang G. Bessler 2018. Experimental investigation of the thermal and cycling behavior of a lithium titanate-based lithium-ion pouch cell. Fournal of Energy Storage 17 (2018), 109 117. DOI: https://doi.org/10.1016/j.est.2018.02.012

[16] Xuebing Han, Minggao Ouyang, Languang Lu, and Jianqiu Li. 2014. Cycle life of commercial lithium-ion batteries with lithium titanium oxide anodes in electric vehicles. Energies 7, 8 (2014), 4895-4909.

[17] Josiah Hester, Lanny Sitanayah, and Jacob Sorber. 2015. Tragedy of the Coulombs: Federating Energy Storage for Tiny, Intermittently-Powered Sensors. In Proceedings of the 13th ACM Conference on Embedded Networked Sensor Systems (SenSys '15). ACM, New York, NY, USA, 5-16. DOI:https://doi.org/10.1145/ 2809695.2809707

[18] Josiah Hester and Jacob Sorber. 2017. Flicker: Rapid Prototyping for the Batteryless Internet-of-Things. In Proceedings of the 15th ACM Conference on Embedded Network Sensor Systems (SenSys '17). ACM, New York, NY, USA, Article 19, 13 pages. DOI : https://doi.org/10.1145/3131672.3131674

[19] Josiah Hester and Jacob Sorber. 2017. The Future of Sensing is Batteryless, Intermittent, and Awesome. In Proceedings of the 15th ACM Conference on Embedded Network Sensor Systems (SenSys '17). ACM, New York, NY, USA, Article 21, 6 pages. DOI : https://doi.org/10.1145/3131672.3131699

[20] Josiah Hester, Kevin Storer, and Jacob Sorber. 2017. Timely Execution on Intermittently Powered Batteryless Sensors. In Proceedings of the 15th ACM Conference on Embedded Network Sensor Systems (SenSys '17). ACM, New York, NY, USA Article 17, 13 pages. DOI : https://doi.org/10.1145/3131672.3131673

[21] HuaHui New Energy. 2013. LTO Battery Specification. http://www.batteryspace com/prod-specs/7455.pdf. (2013).

[22] HuaHui New Energy. 2018. LTO Battery Catalog. https://drive.google.com/file/ d/1TPE2S3EoUMz86eEXSFAPKOOUdnP5ANFD/view. (2018).

[23] Kemet Electronic Components. 2017. FY Series Datasheet. https://content.kemet. com/datasheets/KEM_S6015_FY.pdf. (2017).

[24] Fredrik Larsson and Bengt-Erik Mellander. 2014. Abuse by External Heating, Overcharge and Short Circuiting of Commercial Lithium-Ion Battery Cells. Fournal of The Electrochemical Society 161, 10 (2014), A1611-A1617. http://jes.ecsdl.org/ content/161/10/A1611.abstract

[25] Yoonmyung Lee, Suyoung Bang, Inhee Lee, Yejoong Kim, Gyouho Kim, Mohammad Hassan Ghaed, Pat Pannuto, Prabal Dutta, Dennis Sylvester, and David Blaauw. 2013. A Modular $1 \mathrm{~mm}^{3}$ Die-Stacked Sensing Platform with Low Power $\mathrm{I}^{2} \mathrm{C}$ Inter-die Communication and Multi-Modal Energy Harvesting. In IEEE fournal of Solid-State Circuits (FSSC'13), Vol. 48. Issue 1.

[26] Brandon Lucia, Vignesh Balaji, Alexei Colin, Kiwan Maeng, and Emily Ruppel. 2017. Intermittent Computing: Challenges and Opportunities. In SNAPL.
[27] Alan Mainwaring, David Culler, Joseph Polastre, Robert Szewczyk, and John Anderson. 2002. Wireless sensor networks for habitat monitoring. In Proceedings of the 1st ACM international workshop on Wireless sensor networks and applications. Acm, 88-97.

[28] A. Millner. 2010. Modeling Lithium Ion battery degradation in electric vehicles. In 2010 IEEE Conference on Innovative Technologies for an Efficient and Reliable Electricity Supply. 349-356. DOI : https://doi.org/10.1109/CITRES.2010.5619782

[29] Murata. 2013. Murata Supercapacitor Technical Note. https: www.murata.com/ /media/webrenewal/products/capacitor/edlc/techguide/ electrical/c2m1cxs-053.ashx?la=en. (2013).

[30] Murata. 2016. DMF Series EDLCs. https://www.murata.com/en-us/products/ productdata/8796857270302/MFCDSF1E.pdf?1496719831000. (2016).

[31] Nordic Semiconductor. 2016. NRF52840 Reference Manual. http://infocenter. nordicsemi.com/pdf/nRF52840_PS_v1.0.pdf. (2016).

[32] Noshin Omar, Mohamed Abdel Monem, Yousef Firouz, Justin Salminen, Jelle Smekens, Omar Hegazy, Hamid Gaulous, Grietus Mulder, Peter Van den Bossche, Thierry Coosemans, and Joeri Van Mierlo. 2014. Lithium iron phosphate based battery - Assessment of the aging parameters and development of cycle life model. Applied Energy 113 (2014), 1575 - 1585. DOI : https://doi.org/10.1016/ j.apenergy.2013.09.003

[33] Joseph Polastre, Robert Szewczyk, and David Culler. 2005. Telos: Enabling ultralow power wireless research. In Proceedings of the 4th international symposium on Information processing in sensor networks. IEEE Press, 48.

[34] Samsung Electro-Mechanics. 2013. Multi-layer Ceramic Capacitor. http://www.samsungsem.com/kr/support/product-search/mlcc/_icsFiles/ afieldfile/2017/12/06/Data\%20sheet_CL31A476MOHNNNE.pdf. (2013).

[35] Samsung Electro-Mechanics. 2013. Multi-layer Ceramic Capacitor. http://www. samsungsem.com/kr/front/downloadcms.do?path=/kr/support/product-search/ mlcc/__icsFiles/afieldfile/2014/11/05\&fileName=C_CL32A107MPVNNNE.pdf. (2013).

[36] Sanyo. 2007. Amorphous Silicon Solar Cells / Amorphous Photosensors. https://media.digikey.com/pdf/Data\%20Sheets/Sanyo\%20Energy/ Amorphous_Br.pdf. (2007).

[37] E. Sarasketa-Zabala, I. Gandiaga, E. Martinez-Laserna, L.M. Rodriguez-Martinez, and I. Villarreal. 2015. Cycle ageing analysis of a LiFePO4/graphite cell with dynamic model validations: Towards realistic lifetime predictions. Fournal of Power Sources 275 (2015), 573 - 587. DOI : https://doi.org/10.1016/j.jpowsour.2014. 10.153

[38] Seiko Instruments. 2017. Micro Battery Catalog. https://www.sii.co.jp/en/me/ files/2017/04/MicroBatteryCatalogue-E_2017_web.pdf. (2017).

[39] J. Shen, S. Dusmez, and A. Khaligh. 2013. An advanced electro-thermal cyclelifetime estimation model for LiFePO4 batteries. In 2013 IEEE Transportation Electrification Conference and Expo (ITEC). 1-6.

[40] Shenzhen Hibatt Technology. 2018. Mini LiFePo4 Battery. https:// www.alibaba.com/product-detail/Mini-LiFePO4-battery-10130-30mAh3_60717575578.html?spm=a2700.7724857.main07.8.16295643cyOVQ5. (2018).

[41] Silicon Labs. 2016. SI7021 Datasheet. https://www.silabs.com/documents/public/ data-sheets/Si7021-A20.pdf. (2016).

[42] Joshua R. Smith, Alanson P. Sample, Pauline S. Powledge, Sumit Roy, and Alexander Mamishev. 2006. A Wirelessly-powered Platform for Sensing and Computation. In Proceedings of the 8th International Conference on Ubiquitous Computing (UbiComp'06). Springer-Verlag, Berlin, Heidelberg, 495-506. DOI : https://doi.org/10.1007/11853565_29

[43] M. Swierczynski, D. I. Stroe, A. I. Stan, R. Teodorescu, and S. K. Kœr. 2014. Investigation on the Self-discharge of the $\mathrm{LiFePO} 4 / \mathrm{C}$ nanophosphate battery chemistry at different conditions. In 2014 IEEE Conference and Expo Transportation Electrification Asia-Pacific (ITEC Asia-Pacific). 1-6. DOI : https://doi.org/10.1109/ ITEC-AP.2014.6940762

[44] Texas Instruments. 2015. BQ25505 Datasheet. http://www.ti.com/lit/ds/symlink/ bq25505.pdf. (2015).

[45] TXC. 2010. SMD Seam Sealing Crystals Datasheet. http://www.txccorp.com/ download/products/quartz crystals/2015TXC 7M 17.pdf. (2010).

[46] John Wang, Ping Liu, Jocelyn Hicks-Garner, Elena Sherman, Souren Soukiazian, Mark Verbrugge, Harshad Tataria, James Musser, and Peter Finamore. 2011. Cyclelife model for graphite-LiFePO4 cells. Fournal of Power Sources 196, 8 (2011), 3942 - 3948. DOI : https://doi.org/10.1016/j.jpowsour.2010.11.134

[47] Lohit Yerva, Brad Campbell, Apoorva Bansal, Thomas Schmid, and Prabal Dutta. 2012. Grafting Energy-harvesting Leaves Onto the Sensornet Tree. In Proceedings of the 11th International Conference on Information Processing in Sensor Networks (IPSN '12). ACM, New York, NY, USA, 197-208. DOI: https://doi.org/10.1145/ 2185677.2185733

[48] A. H. Zimmerman. 2004. Self-discharge losses in lithium-ion cells. IEEE Aerospace and Electronic Systems Magazine 19, 2 (Feb 2004), 19-24. DOI : https://doi.org/ 10.1109/MAES.2004.1269687 